\title{
Management des infizierten Implantats im Gesichts-|Schädelbereich
}

Julian Weiser, Christian Lohse, Kilian Kreutzer, Anders Hennigsen, Max Heiland, Alexander Gröbe

\section{Einleitung}

Dentale Implantate sind eine etablierte und risikoarme Therapie bei Zahnverlust in verschiedenen klinischen Situationen geworden. Schätzungsweise werden jedes Jahr 2 Millionen Implantate gesetzt - mit steigender Tendenz aufgrund des demografischen Wandels und der Erfolge der Implantattherapie [1].

Implantate für den Zahnersatz bilden den größten Anteil im Gesichts- und Schädelbereich, in der Mund-, Kieferund Gesichtschirurgie findet jedoch eine Vielzahl weiterer Implantate Anwendung. Bei der Versorgung von Gesichtsschädelfrakturen hat sich die Miniplattenosteosynthese mittels Titanplatten und -schrauben als Standard etabliert. Ist infolge eines ausgeprägten Traumas oder im Rahmen einer Tumorresektion ein Knochendefekt entstanden, so wird dieser i.d.R. durch autologe Fern- oder Nahlappentransplantate oder durch alloplastisches Material in Form von anatomisch vorgeformten oder individuellen Implantaten rekonstruiert. Regionen, in denen es in erster Linie auf eine ästhetische Rehabilitation ankommt (z. B. Ohrmuschel) können mit Epithesen versorgt werden, die über zuvor implantierte Epithesenanker Halt finden. Zuletzt sind in diesem Zusammenhang die Kalottenrekonstruktionen des Neurokraniums zu nennen, die i.d.R. als Defektrekonstruktionen nach Kraniektomien durchgeführt werden. Neben autologen knöchernen und weichgewebigen Fernlappentransplantaten kommen auch hier alloplastische Zemente oder alternativ Titan- oder Kunststoffkonstrukte zum Einsatz.

Insgesamt hat die Implantation von Fremdmaterialien die Therapiemöglichkeiten speziell in der Gesichtsschädelregion substanziell erweitert und ist in der heutigen medizinischen Patientenversorgung unverzichtbar geworden, jedoch kann es im Verlauf zu Komplikationen kommen, von denen die Infektion die häufigste darstellt.

\section{Dentale Implantate}

Bei Beachtung der korrekten Indikation und der individuellen anatomischen Bedingungen gelten dentale Implantate als eine risikoarme Therapie. Nach 16 Jahren Followup konnten Erfolgsraten von 82,9\% gezeigt werden [2].
Entsprechend den Begriffen Gingivitis und Parodontitis für parodontale Entzündungen natürlicher Zähne werden Infektionen des an Implantate angrenzenden Weichund Hartgewebes als Mukositis bzw. Periimplantitis bezeichnet. Mukositis ist ein durch Bakterien verursachter reversibler Entzündungsprozess des periimplantären Weichgewebes, der sich klinisch durch Rötung, Schwellung und Blutung auf Sondieren (bleeding on probing = BOP) äußert. BOP kann ein Indikator für eine periimplantäre Entzündung sein, sein prädiktiver Wert ist bisher jedoch nicht wissenschaftlich belegt. Im Gegensatz zur Mukositis ist die Periimplantitis mit einer irreversiblen Schädigung des angrenzenden Gewebes verbunden und manifestiert sich durch Knochenresorption, reduzierte Osseointegration, erhöhte Taschentiefen und Purulenz [3] (॰ Abb. 1).

Auch wenn die primäre Ätiologie infektiologischer Natur ist, können Risikofaktoren wie Rauchen, schlechte Mundhygiene, vorangegangene Parodontitiden, Diabetes mellitus, genetische Eigenschaften, exzessiver Alkoholkonsum und die Beschaffenheit der Implantatoberfläche zur Entwicklung einer Periimplantitis beitragen $[4,5]$.

\section{Merke}

Die sog. „Zementitis“, ausgelöst durch überschüssigen Zement bei der Eingliederung von Suprakonstruktionen, kann als der häufigste iatrogene Risikofaktor angesehen werden.

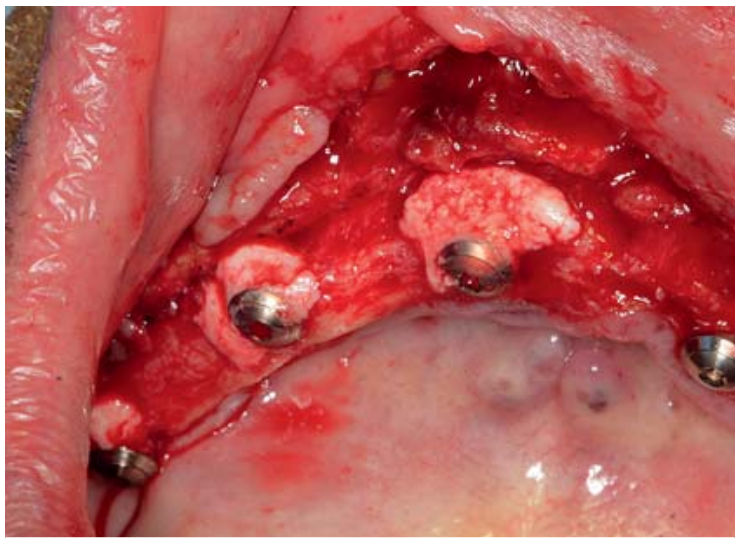

- Abb. 1 Periimplantitis regio 15/16 mit deutlicher Knochenresorption und reduzierter Osseointegration. 
Wilson et al. konnten zeigen, dass in einer Patientengruppe mit klinischen oder radiologischen Zeichen einer Periimplantitis in $81 \%$ Zementrückstände vorhanden waren und dass nach deren Entfernung die entzündlichen Veränderungen in $74 \%$ der betroffenen Areale beseitigt waren [6].

Die durchschnittliche Prävalenz für die periimplantäre Mukositis wird in der Literatur mit $43 \%$ und für die Periimplantitis mit 22\% angegeben [7]. Die Krankheitsbilder gehen fließend ineinander über, wodurch eine klare Abgrenzung erschwert ist. Mögliche diagnostische Kriterien für die Unterscheidung periimplantärer Infektionen sind in $>$ Tab. 1 dargestellt [8].

Die Hauptkriterien für eine korrekte Diagnose wurden in einem Review von Heitz-Mayfield klar definiert [4]. Demzufolge stellen das Sondieren der Taschentiefe (probing depth = PD) und die radiologische Bewertung des Knochenverlusts die vorrangigen diagnostischen Mittel dar. Das Sondieren sollte mit einer Kraft von 0,25 N erfolgen, um periimplantäre Gewebe nicht zu verletzen, und zielt darauf ab, neben der Sondierungstiefe auch den BOP zu erheben, der auf eine entzündliche periimplantäre Mukosa hindeutet. PD und BOP sollten regelmäßig erhoben werden, um ein Fortschreiten der Tiefe über die Zeit, welches i.d.R. mit einem Attachment-Verlust und Knochenabbau einhergeht, rechtzeitig zu erkennen.

Ein festgelegter Grenzwert, ab dem ein entzündlich bedingter marginaler Knochenverlust als „Periimplantitis“ definiert wird, existiert nicht, dieser variiert in der Literatur zwischen $>0,4$ und $>5 \mathrm{~mm}$. Des Weiteren erschwert ein oftmals in den ersten Wochen nach Eingliederung der Suprakonstruktion beobachteter Knochenverlust durch Remodeling, der nicht als Periimplantitis gewertet werden kann, die Diagnostik. Dieser Umstand hat zu der Empfehlung einer Röntgenaufnahme nach Eingliederung der Suprakonstruktion als Ausgangswert für die Beurteilung eines zukünftigen Knochenverlusts geführt.

Als Ausdruck der erschwerten und somit uneinheitlich gehandhabten Abgrenzung der Krankheitsbilder existiert bisher noch keine festgelegte, offizielle Klassifikation der Schweregrade einer Periimplantitis wie es sie für die Parodontitis bereits gibt. Eine mögliche Einteilung wurde 2012 von Froum und Rosen vorgeschlagen [9]. Die Klassifikation basiert auf einer 2-gliedrigen Diagnostik durch Ermittlung der Sondierungstiefe wie auch des Knochenabbaus und teilt die Erkrankung in 3 Schweregrade ein. Der Vorteil dieser Klassifikation ist die Bewertung des relativen Knochenverlusts in Prozent (da jedes Implantat eine andere Länge aufweist), im Gegensatz zum absoluten Verlust in Millimetern ( $\triangleright$ Tab. 2).

Der bakterielle Biofilm auf der Implantatoberfläche spielt eine wichtige Rolle bei der Entwicklung einer Periimplan-
- Tab. 1 Diagnostische Kriterien für periimplantäre Infektionen (Schwarz \& Becker 2015).

\begin{tabular}{|l|l|l|}
\hline & $\begin{array}{l}\text { periimplantäre } \\
\text { Mukositis }\end{array}$ & $\begin{array}{c}\text { Periim- } \\
\text { plantitis }\end{array}$ \\
\hline reversibel & nein & nein \\
\hline $\begin{array}{l}\text { Blutung auf } \\
\text { Sondierung }\end{array}$ & + & + \\
\hline Pus & - & \pm \\
\hline $\begin{array}{l}\text { Zunahme der Son- } \\
\text { dierungstiefen* }\end{array}$ & - & + \\
\hline $\begin{array}{l}\text { Schmerzen } \\
\text { radiologischer } \\
\text { Knochenabbau* }\end{array}$ & - & $(+)$ \\
\hline $\begin{array}{l}\text { Implantatlockerung } \\
\text { mikrobiologische }\end{array}$ & - & + \\
\hline $\begin{array}{l}\text { Tests** } \\
\text { * unspezifisch }\end{array}$ & $\begin{array}{l}\text { unspezi- } \\
\text { felativch zu einem Referenzwert (ideal: Zeitpunkt Einglie- }\end{array}$ \\
\hline $\begin{array}{l}\text { derung der Suprakonstruktion); } \\
\text { tionelle } \text { Testsysteme zur Bestimmung parodontopathogener } \\
\text { Keime }\end{array}$ &
\end{tabular}

- Tab. 2 Klassifikation der Periimplantitis (Froum and Rosen, 2012 [9]).

\begin{tabular}{|c|c|}
\hline Tyр & Charakteristika \\
\hline \multirow[t]{2}{*}{ früh } & $\begin{array}{l}\text { PD } \geq 4 \text { mm (Blutung oder Suppuration bei } \\
\text { Sondierung) }\end{array}$ \\
\hline & Knochenverlust < 25\% der Implantatlänge \\
\hline \multirow[t]{2}{*}{ moderat } & $\begin{array}{l}\mathrm{PD} \geq 6 \mathrm{~mm} \text { (Blutung oder Suppuration bei } \\
\text { Sondierung) }\end{array}$ \\
\hline & $\begin{array}{l}\text { Knochenverlust zwischen } 25 \% \text { und } 50 \% \text { der } \\
\text { Implantatlänge }\end{array}$ \\
\hline \multirow[t]{2}{*}{$\begin{array}{l}\text { fortge- } \\
\text { schritten }\end{array}$} & $\begin{array}{l}\mathrm{PD} \geq 8 \mathrm{~mm} \text { (Blutung oder Suppuration bei } \\
\text { Sondierung) }\end{array}$ \\
\hline & Knochenverlust > 50\% der Implantatlänge \\
\hline
\end{tabular}

titis [10]. Das Management der Periimplantitis basiert auf den gewonnenen Erkenntnissen aus der Behandlung der Parodontitis und zielt auf die Infektions- und Bakterienkontrolle ab. Es wurden sowohl chirurgische als auch nicht chirurgische Therapiemaßnahmen für die Behandlung der Periimplantitis entwickelt.

\section{Konservative Therapie}

Neben der medikamentösen Therapie und der mechanischen Reinigung von Implantatoberflächen mit Küretten, Ultraschall- oder Pulverstrahlsystemen wurden innovative Verfahren wie laserassistierte und photodynamische Therapiemethoden entwickelt. Die mechanische Reinigung kann mit Titan-, Teflon-, Carbon- oder Plastikküretten erfolgen. Da eine Therapie mit konventionellen Küretten die Implantatoberfläche beeinträchtigen und aufrauen kann, wurde für das Arbeitsende der Kürette ein weiche- 
res Material als Titan empfohlen [11]. Sowohl eine Reinigung der Implantatoberflächen mit Carbonhandinstrumenten als auch mit piezoelektrischen Scalern kann den BOP-Index reduzieren, ohne dass zwischen diesen Methoden ein Unterschied im Hinblick auf Sondierungstiefe oder Reduzierung des BOP festgestellt werden konnte allerdings erwies sich im Nachbeobachtungszeitraum von 6 Monaten keine der Methoden als ausreichende Therapie der Periimplantitis [12]. Eine Studie zum Vergleich von Titanküretten mit einem Ultraschallgerät lieferte ähnliche Ergebnisse. Nach 6 Monaten konnten auch hier keine Unterschiede im Hinblick auf die Reduktion der Mikrobiota gezeigt werden [13].

Die Ergebnisse der Pulverstrahlsysteme unterscheiden sich in Abhängigkeit des verwendeteten Mediums und sind signifikant besser in absteigender Reihenfolge: Hydroxylapatit/Tricalciumphosphat $>$ Hydroxylapatit $>$ Glycinpulver $>$ Titandioxid $>$ Wasser und Luft (Kontrollgruppe) > Phosphorsäure [14]. Abrasive Strahlmittel können die Oberfläche von Implantaten modifizieren und bilden Rückstände, wobei das zelluläre Adhäsionsverhalten erhalten bleibt, die Zellantwort verglichen mit sterilen Oberflächen hingegen reduziert ist $[15,16]$. Die Reosseointegrationsrate von Titanimplantaten nach Pulverstrahltherapie wird zwischen 39 und $46 \%$ angegeben, wobei sich das klinische Implantat-Attachment erhöht und die Taschentiefe reduziert [15]. Das Auftreten von Blutungen auf Sondieren, einer der qualitativen Parameter bei Vorliegen einer Periimplantitis, kann signifikant reduziert werden [16].

Der Einsatz lokaler oder systemischer Antibiotika allein ist keine Therapieoption. In Kombination mit anderen konservativen oder chirurgischen Methoden können klinische Periimplantitissymptome jedoch effizienter reduziert werden [17]. Tetrazyklinderivate wie Doxycyclin und Minocyclin bieten den Vorteil, dass sie neben einer antimikrobiellen Wirkung auch durch die Hemmung der Bildung von Matrixmetalloproteinasen einen positiven Effekt auf die bindegewebige Reaktion des Wirtsmechanismus ausüben $[18,19]$. Für die lokale Applikation der Antibiotika sollten diese in geeignete Trägersubstanzen eingebettet sein, die speziell für den Einsatz in der parodontalen Tasche konzipiert wurden. Renvert et al. verglichen in 2 klinischen Studien den Effekt von topischen Minocyclinmikrosphären mit topischem Chlorhexidingel als Ergänzung zur mechanischen Behandlung der Implantatoberfläche [20,21]. Beiden Methoden konnte eine Verbesserung des Blutungsscores und der Taschentiefen nachgewiesen werden, wobei Minocyclinmikrosphären den größeren Einfluss hatten und dieser Effekt auch über 12 Monate erhalten blieb. Im Hinblick auf die bakterielle Besiedlung konnte kein Unterschied der veränderten Bakterienkomposition nachgewiesen werden. Machtei et al. untersuchten Matrixchips auf Gelatinebasis (MatrixC) und chlorhexidinhaltige PerioChips ${ }^{\circledR}$ (PerioC) [22]. Nach
6 Monaten wiederholter Anwendungen konnte zum einen in beiden Gruppen eine signifikante Verbesserung des klinischen Attachmentlevels beobachtet werden und zum anderen war der Effekt der PerioC-Gruppe $(2,19 \pm 0,24 \mathrm{~mm})$ verglichen mit der MatrixC-Gruppe $(1,59 \pm 0,23 \mathrm{~mm})$ signifikant überlegen.

Eine weitere Behandlungsmöglichkeit der Periimplantitis stellt die antimikrobielle photodynamische Therapie (aPDT) dar, die durch Reaktion von Lichtenergie mit einem Photosensibilisator zur Ausbildung von hochreaktiven Sauerstoffspezies führt und somit einen toxischen Effekt auf Mikroorganismen ausübt. In einer Studie wurde diese Methode mit Minocyclinmikrosphären jeweils als ergänzende Therapie zur mechanischen Oberflächenreinigung verglichen [23]. In beiden Gruppen konnte eine ähnlich signifikante Reduktion der Entzündungsreaktion gezeigt werden, sodass auch die aPDT als valide ergänzende Methode in der nicht chirurgischen Therapie der Periimplantitis gewertet werden kann, mit der Einschränkung, dass noch weitere Studien, insbesondere für die Beurteilung des Langzeiteffekts, notwendig sind.

\section{Chirurgische Therapie}

Bei Vorliegen von fortgeschrittenen periimplantären Läsionen mit ausgeprägten Taschentiefen (>5 mm) und Knochenabbau oder nach Versagen konservativer Optionen kann eine chirurgische Therapie indiziert sein. Diese wird in resektive und regenerative Verfahren eingeteilt. Das Prinzip der resektiven Therapie ist die Entfernung der periimplantären knöchernen Defekte durch Osteotomie bzw. Osteoplastik und eine bakterielle Dekontamination durch eine offene Kürettage. Zusätzlich kann durch Glätten und Polieren der Implantatoberfläche eine sog. Implantoplastik durchgeführt werden. Die regenerative Therapie hingegen strebt eine vollständige Regeneration und Reosseointegration an, indem knöcherne periimplantäre Defekte nach Entfernung des entzündlichen Gewebes mit Knochenersatzmaterial oder Knochentransplantat aufgefüllt werden. Zusätzlich kann dieser Bereich durch Einbringen einer Membran, im Sinne einer gesteuerten Knochenregeneration (GBR), von dem rascher regenerierenden Weichgewebe abgeschirmt werden, um eine ungestörte knöcherne Heilung zu ermöglichen.

Eine aktuelle Metaanalyse konnte zeigen, dass mit beiden Therapieansätzen eine signifikante Reduktion des marginalen Knochenverlusts möglich ist [24]. Dennoch bietet die Datenlage keine sicheren oder spezifischen Empfehlungen für die chirurgische Therapie der Periimplantitis [25]. Serino et al. zeigten in einer Studie, dass ein resezierendes Vorgehen durch Taschenelimination und Knochenrekonturierung in Verbindung mit Plaquekontrolle vor und nach dem Eingriff eine effektive Therapie der Periimplantitis darstellt [26]. Der zusätzliche Nutzen einer Implantoplastik als Ergänzung zu resezierendem chirurgischem Vorgehen wurde durch Romeo et al. in 
einer radiologischen Studie untersucht [27]. In der Nachbeobachtungszeit von 3 Jahren konnte in der Studiengruppe ein geringerer Knochenverlust als nach ausschließlich resektiver Therapie nachgewiesen werden. Die Implantoplastik zeigte nach 24 Monaten auch positive Effekte auf klinische Parameter wie Taschentiefe, Attachmentlevel und Blutungsindizes [28].

\section{Merke}

Die resektive chirurgische Therapie in Form von Osteotomie und Osteoplastik, insbesondere in Verbindung mit einer Implantoplastk, stellt eine valide und effektive Behandlungsoption der Periimplantitis dar. Aufgrund der postoperativ häufigeren gingivalen Rezessionen ist dieses Vorgehen jedoch nicht in jeder Situation das richtige Vorgehen, dies gilt insbesondere im Hinblick auf ästhetisch anspruchsvolle Areale [29].

Hinsichtlich des regenerativ-chirurgischen Ansatzes können autologe, allogene oder xenogene Knochenersatzmaterialien für die Auffüllung des Defekts verwendet werden. Gegebenenfalls kann dieser Bereich vor einwachsendem Bindegewebe durch eine resorbierbare oder nicht resorbierbare Membran geschützt werden. Allogenes und xenogenes Material kann als fast gleichwertig zu autogenem Knochen angesehen werden [30,31], der oft als der „Goldstandard“ bezeichnet wurde. Ein wesentlicher Unterschied ist, dass autogener Knochen ungefähr $40 \%$ Volumenverlust in der Heilungsphase aufweist, während hingegen synthetische Knochenersatzmaterialien eine hohe Volumenstabilität besitzen, jedoch selbst Jahre nach dem Eingriff kaum resorbiert werden [32]. Es gibt Hinweise, dass autologer Knochen die Knochenneubildung weder fördert noch hemmt, wodurch der ehemalige „Goldstandard“ infrage gestellt wird [33].

Dem Behandler steht eine Vielzahl an alternativen Materialien auf dem Markt zur Verfügung, auf die im Rahmen dieses Artikels nicht allumfassend eingegangen werden kann. Eine wesentliche Studie zum Vergleich von alloplastischem mit xenogenem Material für die Defektauffüllung im Rahmen der Periimplantitistherapie wurde von Schwarz et al. durchgeführt.

Das 2-Jahres-Ergebnis konnte sowohl nanokristallinem Hydroxylapatit als auch natürlichem Knochenmineral bovinen Ursprungs in Kombination mit einer Kollagenmembran eine klinisch signifikante Reduktion der Taschentiefe und einen Zugewinn an Attachment nachweisen [34]. Nach 4 Jahren stellte sich allerdings die Anwendung von natürlichem Knochenmineral in Verbindung mit einer Kollagenmembran als die effektivere Methode zur regenerativen Behandlung der Periimplantitis dar [35]. Ob eine Membran in diesem Zusammenhang einen positiven Effekt auf die Heilung ausübt, lässt sich anhand der Studie nicht beurteilen, da unterschiedliche Ersatzmaterialien

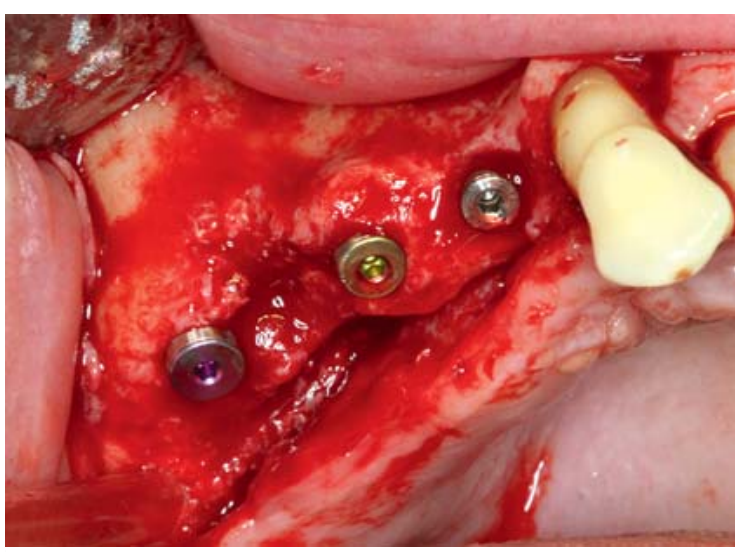

- Abb. 2 Regenerativ-chirurgisch therapierte Periimplantitis regio 32-36. Im Anschluss an eine intensive Oberflächenreinigung wurde der Defekt mit natürlichem Knochenmineral aufgefüllt.

verglichen wurden. Ein Beispiel für eine regenerativ-chirurgisch therapierte Periimplantitis ist in $\mathbf{A b b} \mathbf{2}$ dargestellt.

Den Großteil chirurgisch-regenerativ therapierter Periimplantitisfälle stellt die GBR unter Verwendung von Knochenersatzmaterial mit einer Membran dar [36]. Der Einsatz nicht resorbierbarer PTFE-Membranen im Rahmen der GBR zeigte keine signifikanten Unterschiede im Hinblick auf die Reosseointegration im Vergleich zur Behandlung ohne diese Membran [37]. Neueste Studien mit z. T. 5-Jahres-Nachbeobachtungsintervall konnten selbst den häufig verwendeten resorbierbaren Membranen keinen fundamentalen Vorteil nachweisen [38, 39].

Merke

Sollten alle therapeutischen Maßnahmen scheitern, kann die fortschreitende Periimplantitis zur Implantatlockerung führen. In diesen Fällen ist die Entfernung des Implantats mit einem anschließenden regenerativen Vorgehen die einzige Therapieoption $[28,40,41]$.

\section{Osteosynthesematerial}

Auf diese Thematik wurde bereits eingehend in dem Artikel „Materialentfernung in der kraniomaxillofazialen Traumatologie“, erschienen im OP-journal Ausgabe 2/2016 eingegangen. Zu den häufigsten Ursachen von Frakturen des Gesichtsschädels zählen Verkehrsunfälle, Stürze, Körperverletzungsdelikte und Sportverletzungen. Die Indikation zur operativen Versorgung wird in Abhängigkeit von der anatomischen Lage der Fraktur sowie vom Dislokationsgrad und der klinischen Symptomatik gestellt. Im Gesichtsschädelbereich stellt die Osteosynthese durch Open Reduction internal Fixation (ORIF) die operative 
Therapie der Wahl dar. Die häufigsten Komplikationen sind Materiallockerung, freiliegendes Osteosynthesematerial oder Infektion [42-44] ( Abb. 3).

Klinische Symptome infizierten Osteosynthesematerials sind klassische Entzündungszeichen wie Rötung, Schwellung, Schmerz und Überwärmung. Auch Fieber und erhöhte laborchemische Entzündungsparameter können auftreten. Das Débridement nekrotischer Gewebeanteile und eine antibiotische Therapie sind unumstritten. Klare Empfehlungen, ob infiziertes Material entfernt werden muss, und wenn es entfernt werden muss, ob es direkt durch erneute ORIF ersetzt werden kann, gibt es nicht [45-47]. Der Gesichtsschädelbereich wird aufgrund seiner guten Durchblutung durch Kollateralgefäße im Vergleich zu anderen Regionen, wie z.B. den Extremitäten, als privilegierter Bereich angesehen, sodass viele Autoren eine Materialentfernung nicht als zwingend erforderlich ansehen [45,47-49]. Eine Metaanalyse von Cahill et al., die 7503 Patienten mit Osteosynthesematerial im Gesichtsschädelbereich einschloss, zeigte eine Inzidenz der Infektion von 6,8\% [50]. Bei diesen Infektionen wurde das Material in 51,8\% der Fälle entfernt, in 32,6\% der Fälle in situ belassen und 15,6\% der Fälle wurde das Vorgehen nicht berichtet.

\section{Merke}

Insgesamt hatte das Entfernen oder Belassen des Osteosynthesematerials keinen signifikanten Einfluss auf das Behandlungsergebnis.

In der gleichen Studie wird ein evidenzbasierter Therapiealgorithmus für das Management des infizierten Osteosynthesematerials im Gesichtsschädelbereich vorgestellt. Demnach ist, nach Ausschluss einer Osteomyelitis oder Pseudarthrose, die Bruchspaltkonsolidierung entscheidend für das weitere Vorgehen. Sind die Frakturenden suffizient angenähert, die laborchemischen Entzündungsparameter normwertig und die Knochenverhältnisse stabil, muss das Material nicht entfernt werden. Bei Vorliegen einer klinisch oder radiologisch nachweisbaren deutlichen Lücke zwischen den Frakturenden, weichen Knochenverhältnissen oder erhöhten Infektparametern empfehlen die Autoren, neben einer antibiotischen Therapie, die Entfernung des Osteosynthesematerials, Débridement von nekrotischem Knochen, erneute ORIF und Knochenaufbau ( $\bullet$ Abb. 4).

Der Grenzbereich stellt die größte Herausforderung im Hinblick auf die Entscheidungsfindung dar. Bei Frühinfekten sollte das Material belassen werden, in fortgeschritteneren Phasen sollte das Material ausgewechselt werden und die medizinischen Rahmenbedingungen, z. B. durch Sistieren von Tabakprodukten, verbessert werden. Intraoperativ sollten Abstriche genommen werden, um eine zunächst kalkulierte antibiotische Therapie im Verlauf resistenzgerecht umzustellen.

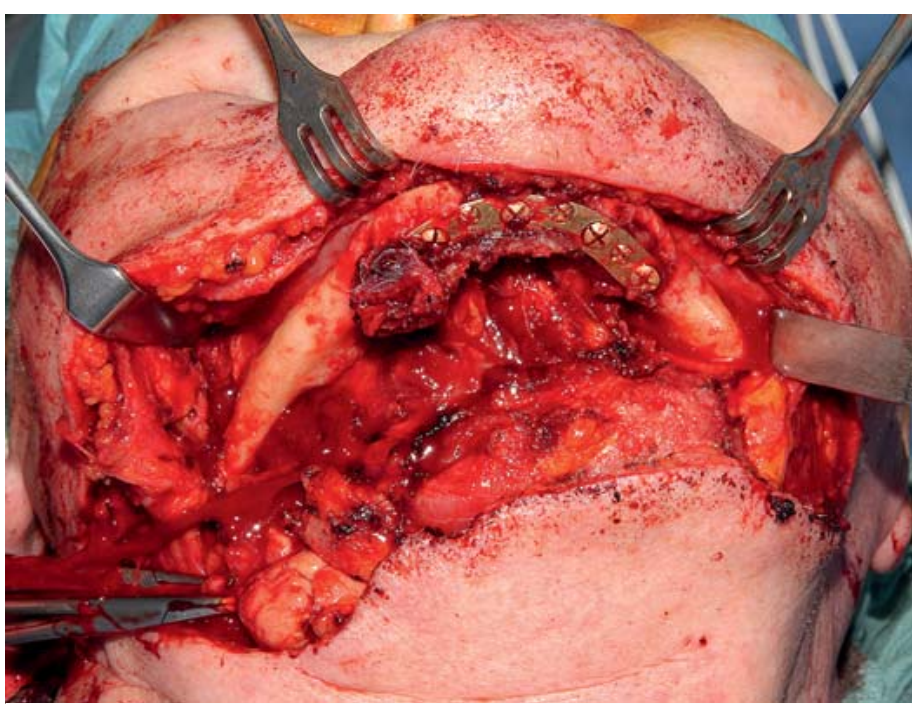

- Abb. 3 Infiziertes Osteosynthesematerial im Bereich des anterioren Unterkiefers.

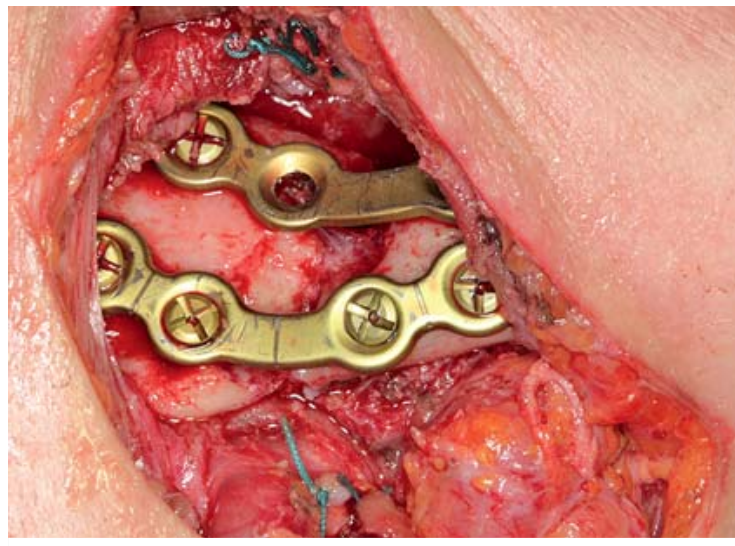

- Abb. 4 Infiziertes Osteosynthesematerial im Bereich des rechten Kieferwinkels mit ausbleibender Bruchspaltkonsolidierung.

\section{Merke}

Eine externe Fixation im Gesichtsschädelbereich ist theoretisch möglich und wurde bereits angewandt, sie ist in diesem Bereich jedoch unpraktisch in der Durchführung, hat einen verheerenden Einfluss auf die Lebensqualität und ist vor allem nicht notwendig [50].

\section{Kranioplastik}

Die Kranioplastik zählt zu den ältesten chirurgischen Eingriffen. Infolge schwerer Traumata, nach Tumorresektion oder zur Reduktion des intrakraniellen Druckes kann ein Knochendefekt des Neurokraniums entstehen. Dieser Defekt kann durch eine Kranioplastik mit autologem Kno- 


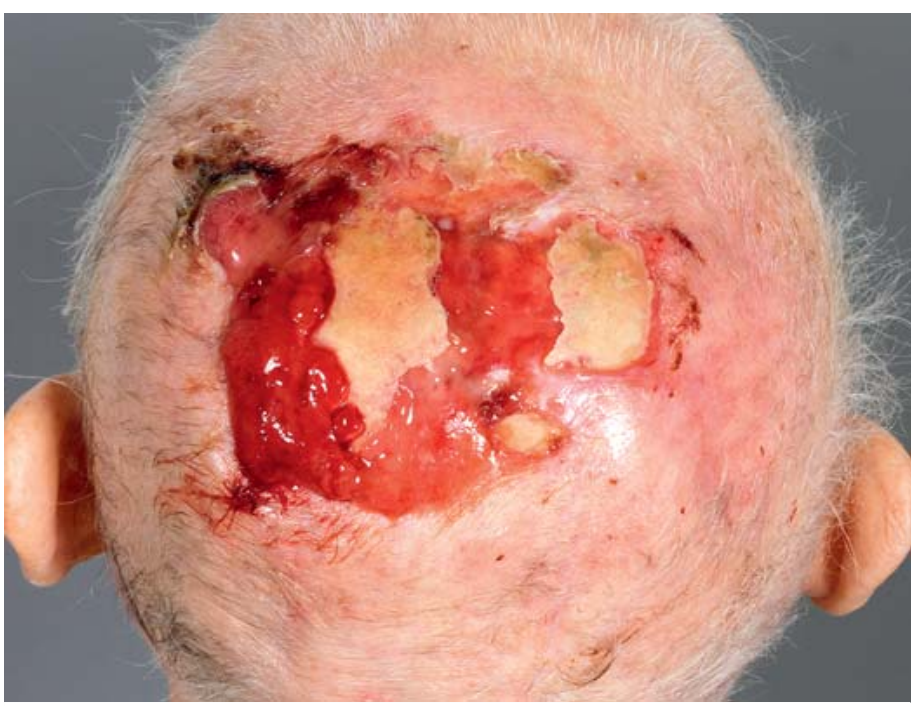

- Abb. 5 Atypisches Fibroxanthom der Kopfhaut - präoperativ.

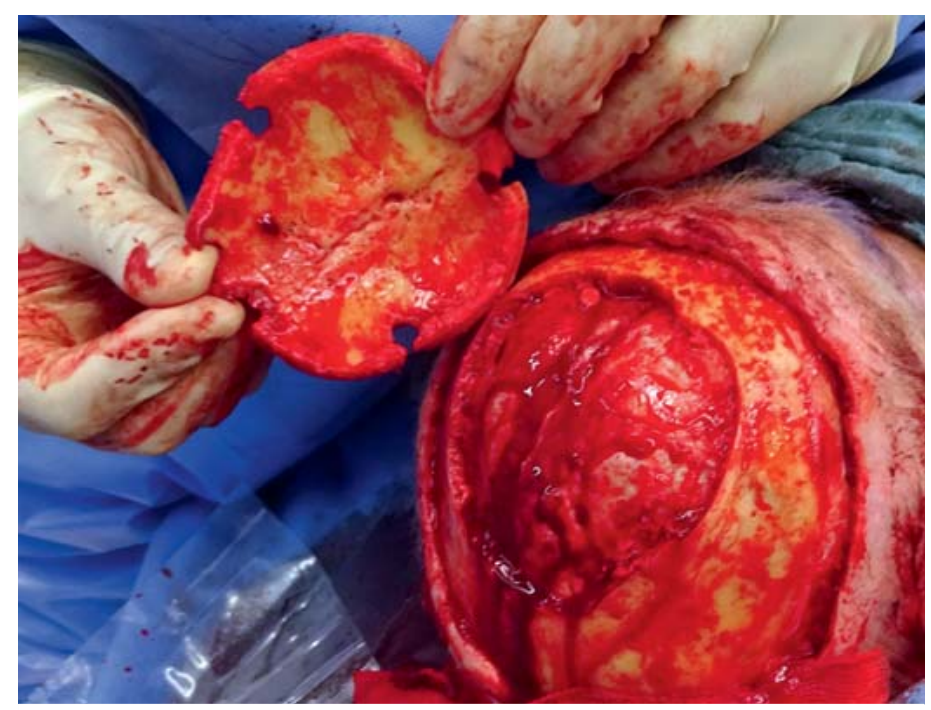

- Abb. 6 Tumorresektion durch schonende Entfernung eines Knochendeckels.

chen oder mit synthetischem Material rekonstrukiert werden. Sowohl die Wahl des Materials als auch der optimale Zeitpunkt des Eingriffs wird bis dato intensiv und zwiespältig diskutiert. Neben autologem Knochenersatz stehen Titannetze, Polymethylmethacrylat (Palacos ${ }^{\circledR}$ ) oder bei größeren Defekten auch im CAD-/CAM-Verfahren (CAD: Computer aided Design/CAM: Computer aided Manufacturing) gefertigte Implantate aus Titan oder Hydroxylapatit zur Verfügung. Aufgrund von Immunkompatibilität, Infektionsresistenz und der Stimulation lokaler Knochenregeneration wird der patienteneigene Knochen als das Material der ersten Wahl für eine Kranioplastik angesehen. Andererseits wird der transplantierte Knochen leicht resorbiert, birgt eine höhere Entnahmemorbidität als alloplastische Verfahren und steht nur in begrenztem Ausmaß, insbesondere für größere Defekte, zur Verfügung [51]. Neben der Entnahmemorbidität bestehen weitere Vorteile der synthetischen Biomaterialien in kürzeren Operationszeiten und Krankenhausaufenthalten [52].

Alle Rekonstruktionsmaterialien haben ihre Vor- und Nachteile, wobei keines alle Kriterien für das ideale Implantat erreicht. Ein Beispiel für die Anwendung alloplastischen Materials für die Kranioplastik stellt der folgende Case Report aus unserer Klinik dar, in welchem aufgrund einer ausgedehnten Tumorresektion der Kalotte mit ausreichendem Sicherheitsabstand eine Rekonstruktion mit patienteneigenem Gewebe nicht zu realisieren war.

\section{CASE REPORT}

Die 76-jährige Patientin wurde uns zur Weiterbehandlung eines histologisch gesicherten und mehrfach voroperierten atypischen Fibroxanthoms im Bereich der parietookzipitalen Kopfhaut vorgestellt. Bei der Erstvorstellung in unserer Abteilung zeigte sich bereits klinisch eine Tumorinfiltration der Kalotte ( $\triangleright$ Abb. 5). In der MRT kam keine Affektion der Dura zur Darstellung. Es folgte eine CAD-/CAM-Planung zur geführten Resektion der Kalotte und Rekonstruktion mit einem Titanimplantat. In Zusammenarbeit mit den Kollegen der Neurochirurgie wurde die Tumorresektion mit ausreichendem Sicherheitsabstand vorgenommen ( $\bullet$ Abb. 6). Die Rekonstruktion der Kalotte und der Kopfhaut wurde mit dem patientenspezifischen Titanimplantat $(\triangleright \mathbf{A b b}$. 7) und einem myokutanen Latissimus-dorsi-Transplantat erzielt. In der abschließenden histopathologischen Untersuchung kam ein teils spindel-, teils pleomorphzelliges Sarkom mit tumorfreien Resektionsrändern zur Darstellung. Nach intensivmedizinischer Überwachung konnte die Patienten mit stadiengerechten Wundverhältnissen und einem neurologisch unauffälligen Status auf die kieferchirurgische Normalstation übernommen werden ( $\bullet$ Abb. 8).

Zu den häufigsten Komplikationen des Rekonstruktionseingriffs einer Kranioplastik zählt die Infektion, deren Inzidenz, je nach Studie, zwischen 6 und 16\% angegeben wird [53]. In einer aktuellen Studie von Kimchi et al. sind in Kulturen von Wundabstrichen in absteigender Reihenfolge Staphylococcus aureus, Propionibakterien, Klebsiella pneumoniae und Enterobacter aerogenes zu finden [53]. In der Regel war intravenös appliziertes Cefazolin das Antibiotikum der Wahl, ohne die Infektionsrate signifikant zu reduzieren. Dies steht im Kontrast zu dem Ergebnis einer Studie von Young et al., in der eine perioperative Antibiotikaprophylaxe im Rahmen von neurochirurgischen Operationen die Infektionsrate von 3,64\% in 
der Kontrollgruppe auf 0,96\% in der Studiengruppe mit antibiotischer Prophylaxe signifikant reduzierte [54].

Prädiktive Parameter, um die Entstehung einer Infektion verhindern zu können, wurden häufig untersucht - mit z.T. inkongruenten Ergebnissen. Walcott et al. fanden Hinweise dass Voroperationen und Schlaganfall als Indikation für den Eingriff signifikant mit der Entwicklung von Kranioplastikinfektionen assoziiert sind [55]. Patientenalter, Lokalisation, Material und Zeitpunkt hatten keinen Einfluss auf die Infektionsrate. Malcolm et al. untersuchten insbesondere den Einfluss des Zeitpunkts des Eingriffs auf die Komplikationsrate [56]. Hierbei barg die frühe Kranioplastik (<90 Tage) zwar ein höheres Risiko für die Entwicklung eines Hydrozephalus, insbesondere in der Traumagruppe, bez. anderer Komplikationen fanden sich jedoch keine Unterschiede zur späten Kranioplastik (>90 Tage).

Eine Metaanalyse von Yadla et al. hingegen konnte weder zwischen früher und später Kranioplastik noch zwischen autologem Knochen und allplastischem Material noch zwischen subkutaner oder extrakorporaler Aufbewahrung des Knochentransplantats einen signifikanten Unterschied im Hinblick auf die Infektionsrate nachweisen [57].

Die Literatur bez. fremdmaterialassoziierter Infektionen in der Neurochirurgie ist rar, ohne dass standardisierte Behandlungskonzepte existieren.

\footnotetext{
Merke

In der Regel ist ein erneuter chirurgischer Eingriff in Kombination mit einer ausgedehnten antimikrobiellen Antibiofilmtherapie von meist 12 Wochen notwendig [58].
}

Es gibt jedoch Hinweise, dass einige Patientengruppen von Behandlungsalgorithmen ohne Entfernung oder mit einem einzeitigen Wechsel des Implantats profitieren können [58]. Damit in Übereinstimmung konnten Brown et al. zeigen, dass die Erfolgsrate einer konservativen Therapie durch eine Kombination von intravenöser mit intrathekaler Antibiotikatherapie, selbst bei Liquor-Shunt-Infektionen durch koagulasenegative Staphylokokken, vergleichbar mit einem konventionellen Management im Sinne einer Explantation mit Antibiotikatherapie ist [59].

\section{Schlussfolgerungen}

Implantate im Gesichtsschädelbereich sind insgesamt als risikoarm zu werten, die häufigste Komplikation ist jedoch die Infektion. Es existieren weder für den Bereich dentaler Implantate oder des Osteosynthesematerials noch für die Kranioplastik standardisierte Therapieempfehlungen für fremdmaterialassoziierte Infektionen. So-

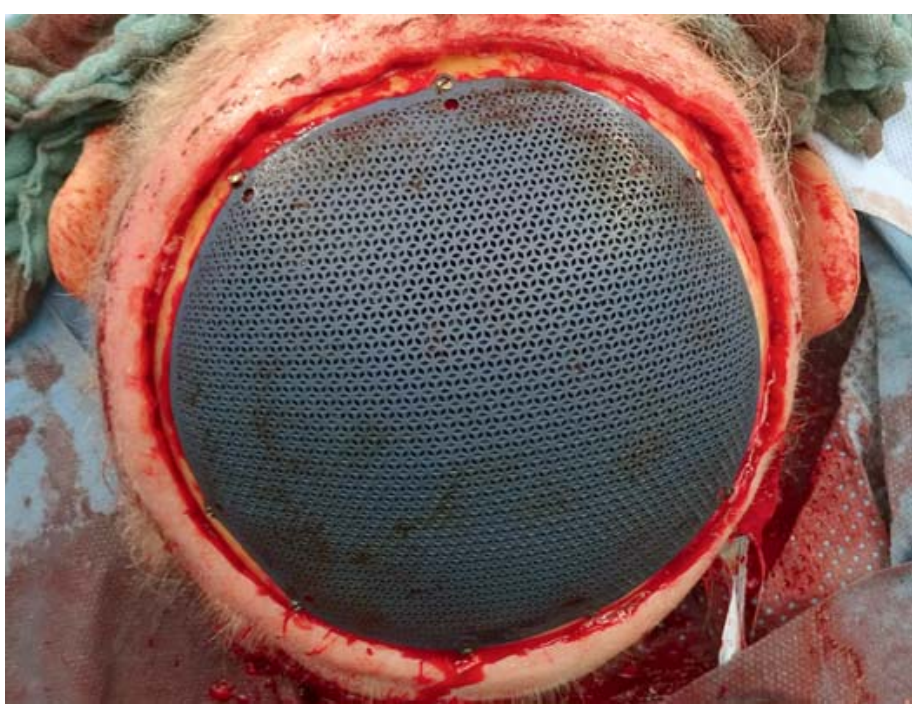

- Abb. 7 Rekonstruktion des knöchernen Defekts mit einem patientenspezifischen Titanimplantat.

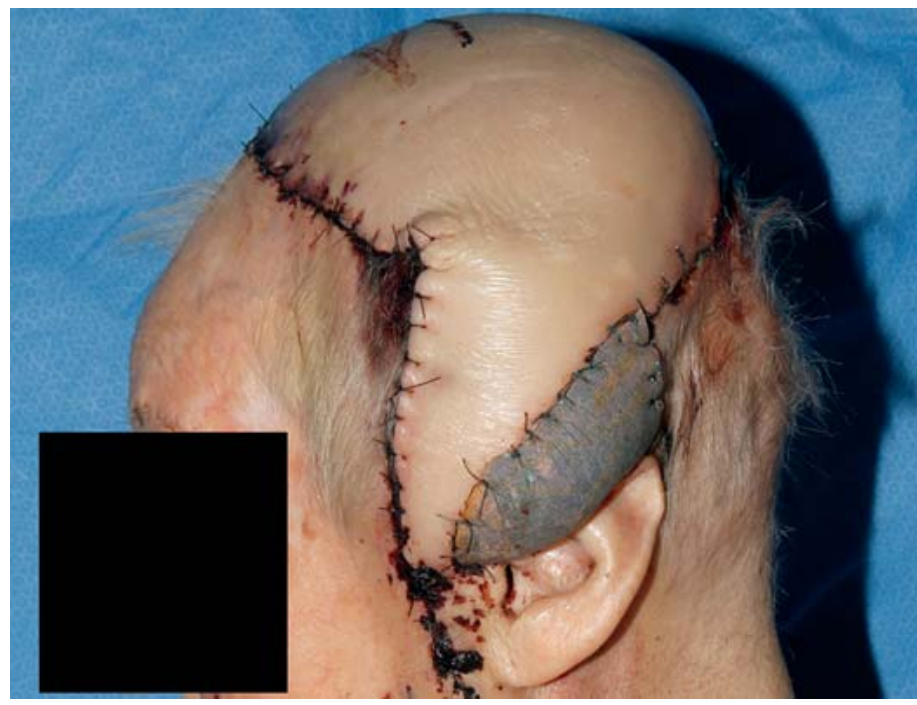

- Abb. 8 Rekonstruktion des häutigen Defekts mit einem myokutanen Latissimus-dorsi-Transplantat. Angrenzend an die Ohrmuschel ist ein Anteil Kunsthaut sichtbar.

mit ist die spezielle Behandlungsplanung stark von der Erfahrung und Einschätzung der individuellen Pathologie durch den Behandler abhängig.

Die frühe Periimplantitis im dentalen Bereich kann konservativ durch mechanische Oberflächenreinigung behandelt werden, in fortgeschrittenen Stadien kann eine chirurgische Therapie notwendig werden - als Anhaltspunkt für einen Grenzwert kann eine Sondierungstiefe von $5 \mathrm{~mm}$ gelten. Sowohl die resektive als auch die regenerative chirurgische Therapie stellen valide Behandlungsoptionen dar, wobei ein resezierendes Vorgehen 
aus ästhetischen Gründen eher dem nicht sichtbaren Seitenzahngebiet vorbehalten ist. Regenerative Chirurgie in Verbindung mit einer resorbierbaren Membran im Sinne der GBR ist eine gängige Therapie. Weitere Studien sind notwendig, um sich sowohl Klarheit über Vorteile der unterschiedlichen Knochenersatzmaterialien und Membranen auf dem Markt zu verschaffen als auch, um den Nutzen adjuvanter innovativer Verfahren zu bewerten sowie Langzeitergebnisse zu liefern.

Merke

Im Vergleich zu anderen anatomischen Regionen, wie z. B. den Extremitäten, muss infiziertes Osteosynthesematerial im Gesichtsschädelbereich durch die hervorragende Gewebeperfusion nicht zwangsläufig entfernt werden

Entscheidend ist hier die Bruchspaltkonsolidierung. Gibt es Hinweise auf eine fehlende knöcherne Konsolidierung, sollte das infizierte Material entfernt werden und die Frakturenden nach Débridement durch erneute ORIF approximiert und versorgt werden. Eine zunächst kalkulierte antibiotische Therapie sollte nach Erhalt des Antibiogramms resistenzgerecht adaptiert werden.

Auch für die Kranioplastik mit Fremdmaterial in der Neurochirurgie stellt die Infektion die häufigste Komplikation dar. In vielen Studien wurden auslösende Faktoren untersucht, um die Entstehung einer Infektion zu vermeiden. Insgesamt konnte die Meinung, dass eine frühe Kranioplastik Infektionen begünstigt, nicht bestätigt werden. Umso geringer ist die Studienlage im Hinblick auf das Vorgehen bei manifester Infektion. Aufgrund der dramatischen Konsequenzen durch die Nähe zum Liquorraum wird das infizierte Fremdmaterial i.d.R. entfernt. Einige Autoren liefern Hinweise auf eine mögliche erfolgreiche konservative Therapie, auch hier sind jedoch weitere Studien mit größeren Patientengruppen notwendig, um die Sicherheit und Indikation dieses Vorgehens zu bewerten, sowie um einen genauen Behandlungsalgorithmus festzulegen.

\section{Interessenkonflikt}

Die Autoren bestätigen keinen Interessenkonflikt für o. a. Manuskript.

\section{Über die Autoren}

\section{Julian Weiser}

Dr. med. dent., Wissenschaftlicher Mitarbeiter, Klinik und Poliklinik für Mund-, Kiefer- und Gesichtschirurgie, Universitätsklinikum Hamburg-Eppendorf

\section{Christian Lohse}

Dr. med., Leitender Oberarzt, Klinik für Mund-, Kiefer-, Gesichtschirurgie, Diakovere Henriettenstift gGmbH

\section{Kilian Kreutzer}

Dr. med. Dr. med. dent., Oberarzt, Klinik und Poliklinik für Mund-, Kiefer- und Gesichtschirurgie, Charité - Universitätsmedizin Berlin

\section{Anders Hennigsen}

Dr. med. Dr. med. dent., Wissenschaftlicher Mitarbeiter, Klinik und Poliklinik für Mund-, Kiefer- und Gesichtschirurgie, Universitätsklinikum Hamburg-Eppendorf

\section{Alexander Gröbe}

Priv.-Doz. Dr. med. Dr. med. dent., Chefarzt, Klinik für Mund-, Kiefer-, Gesichtschirurgie, Diakovere Henriettenstift gGmbH

\section{Max Heiland}

Prof. Dr. med. Dr. med. dent., Klinikdirektor, Klinik und Poliklinik für Mund-, Kiefer- und Gesichtschirurgie, Charité Universitätsmedizin Berlin

\section{Korrespondenzadresse}

Priv.-Doz. Dr. med. Dr. med. dent. Alexander Gröbe

Klinik für Mund-, Kiefer-, Gesichtschirurgie

Diakovere Henriettenstift gGmbH

Marienstr. 72-90

30171 Hannover

alexander.groebe@diakovere.de

\section{Literatur}

[1] Klinge B, Hultin M, Berglundh T. Peri-implantitis. Dent Clin North Am 2005; 49: 661-676

[2] Simonis P, Dufour T, Tenenbaum H. Long-term implant survival and success: a 10-16-year follow-up of non-submerged dental implants. Clin Oral Implants Res 2010; 21: 772-777

[3] Khammissa RA, Feller L, Meyerov R et al. Peri-implant mucositis and peri-implantitis: clinical and histopathological characteristics and treatment. SADJ 2012; 67: 122, 124-126

[4] Heitz-Mayfield LJ. Peri-implant diseases: diagnosis and risk indicators. J Clin Periodontol 2008; 35 (8 Suppl.): S292-S304

[5] Heitz-Mayfield L], Huynh-Ba G. History of treated periodontitis and smoking as risks for implant therapy. Int J Oral Maxillofac Implants 2009; 24 (Suppl.): S39-S68

[6] Wilson TG jr. The positive relationship between excess cement and peri-implant disease: a prospective clinical endoscopic study. J Periodontol 2009; 80: 1388-1392

[7] Derks J, Tomasi C. Peri-implant health and disease. A systematic review of current epidemiology. J Clin Periodontol 2015; 42 (Suppl. 16): S158-S171

[8] Schwarz FB, Becker J. Periimplantäre Infektionen. Ein Update zur Epidemiologie, Ätiologie, Diagnostik, Prävention und Therapie. Implantologie 2015; 23: 247-259

[9] Froum S], Rosen PS. A proposed classification for peri-implantitis. Int J Periodontics Restorative Dent 2012; 32: 533-540

[10] Canullo L, Penarrocha-Oltra D, Covani U et al. Microbiologic and clinical findings of implants in healthy condition and with peri-implantitis. Int J Oral Maxillofac Implants 2015; 30: 834842 
[11] Unursaikhan O, Lee JS, Cha JK et al. Comparative evaluation of roughness of titanium surfaces treated by different hygiene instruments. J Periodontal Implant Sci 2012; 42: 88-94

[12] Karring ES, Stavropoulos A, Ellegaard B et al. Treatment of peri-implantitis by the Vector system. Clin Oral Implants Res 2005; 16: 288-293

[13] Persson GR, Samuelsson E, Lindahl C et al. Mechanical nonsurgical treatment of peri-implantitis: a single-blinded randomized longitudinal clinical study. II. Microbiological results. J Clin Periodontol 2010; 37: 563-573

[14] Tastepe CS, Liu Y, Visscher CM et al. Cleaning and modification of intraorally contaminated titanium discs with calcium phosphate powder abrasive treatment. Clin Oral Implants Res 2013; 24: 1238-1246

[15] Tastepe CS, van Waas R, Liu Y et al. Air powder abrasive treatment as an implant surface cleaning method: a literature review. Int J Oral Maxillofac Implants 2012; 27: 1461-1473

[16] Sahm N, Becker J, Santel T et al. Non-surgical treatment of peri-implantitis using an air-abrasive device or mechanical debridement and local application of chlorhexidine: a prospective, randomized, controlled clinical study. J Clin Periodontol 2011; 38: 872-878

[17] Muthukuru M, Zainvi A, Esplugues EO et al. Non-surgical therapy for the management of peri-implantitis: a systematic review. Clin Oral Implants Res 2012; 23 (Suppl. 6): S77-S83

[18] Grenier D, Plamondon P, Sorsa T et al. Inhibition of proteolytic, serpinolytic, and progelatinase-b activation activities of periodontopathogens by doxycycline and the non-antimicrobial chemically modified tetracycline derivatives. J Periodontol 2002; 73: 79-85

[19] Park JB. Effects of doxycycline, minocycline, and tetracycline on cell proliferation, differentiation, and protein expression in osteoprecursor cells. J Craniofac Surg 2011; 22: 1839-1842

[20] Renvert S, Lessem J, Dahlen G et al. Topical minocycline microspheres versus topical chlorhexidine gel as an adjunct to mechanical debridement of incipient peri-implant infections: a randomized clinical trial. J Clin Periodontol 2006; 33: 362-369

[21] Renvert S, Lessem J, Dahlen G et al. Mechanical and repeated antimicrobial therapy using a local drug delivery system in the treatment of peri-implantitis: a randomized clinical trial. J Periodontol 2008; 79: 836-844

[22] Machtei EE, Frankenthal S, Levi G et al. Treatment of peri-implantitis using multiple applications of chlorhexidine chips: a double-blind, randomized multi-centre clinical trial. J Clin Periodontol 2012; 39: 1198-1205

[23] Bassetti M, Schar D, Wicki B et al. Anti-infective therapy of peri-implantitis with adjunctive local drug delivery or photodynamic therapy: 12-month outcomes of a randomized controlled clinical trial. Clin Oral Implants Res 2014; 25: 279-287

[24] Ramanauskaite A, Daugela P, Juodzbalys G. Treatment of periimplantitis: meta-analysis of findings in a systematic literature review and novel protocol proposal. Quintessence Int 2016; 47: 379-393

[25] Mombelli A, Moëne R, Decaillet F. Surgical treatments of periimplantitis. Eur J Oral Implantol 2012; 5 (Suppl.): S61-S70

[26] Serino G, Turri A. Outcome of surgical treatment of peri-implantitis: results from a 2-year prospective clinical study in humans. Clin Oral Implants Res 2011; 22: 1214-1220

[27] Romeo E, Lops D, Chiapasco M et al. Therapy of peri-implantitis with resective surgery. A 3-year clinical trial on rough screw-shaped oral implants. Part II: radiographic outcome. Clinical Oral Implants Res 2007; 18: 179-187
[28] Romeo E, Ghisolfi M, Murgolo $\mathrm{N}$ et al. Therapy of peri-implantitis with resective surgery. A 3-year clinical trial on rough screw-shaped oral implants. Part I: clinical outcome. Clinical Oral Implants Res 2005; 16: 9-18

[29] Smeets R, Henningsen A, Jung O et al. Definition, etiology, prevention and treatment of peri-implantitis - a review. Head Face Med 2014; 10: 34

[30] Kolk A, Handschel J, Drescher W et al. Current trends and future perspectives of bone substitute materials - from space holders to innovative biomaterials. J Craniomaxillofac Surg 2012; 40: 706-718

[31] Fischer J, Kolk A, Wolfart $S$ et al. Future of local bone regeneration - protein versus gene therapy. J Craniomaxillofacial Surg 2011; 39: 54-64

[32] lezzi G, Degidi M, Scarano A et al. Anorganic bone matrix retrieved 14 years after a sinus augmentation procedure: a histologic and histomorphometric evaluation. J Periodontol 2007; 78: 2057-2061

[33] Araujo MG, Lindhe J. Socket grafting with the use of autologous bone: an experimental study in the dog. Clin Oral Implants Res 2011; 22: 9-13

[34] Schwarz F, Sculean A, Bieling K et al. Two-year clinical results following treatment of peri-implantitis lesions using a nanocrystalline hydroxyapatite or a natural bone mineral in combination with a collagen membrane. J Clin Periodontol 2008; 35 : 80-87

[35] Schwarz F, Sahm N, Bieling K et al. Surgical regenerative treatment of peri-implantitis lesions using a nanocrystalline hydroxyapatite or a natural bone mineral in combination with a collagen membrane: a four-year clinical follow-up report. J Clin Periodontol 2009; 36: 807-814

[36] Sahrmann P, Attin T, Schmidlin PR. Regenerative treatment of peri-implantitis using bone substitutes and membrane: a systematic review. Clin Implant Dent Relat Res 2011; 13: 46-57

[37] Wetzel AC, Vlassis J, Caffesse RG et al. Attempts to obtain reosseointegration following experimental peri-implantitis in dogs. Clin Oral Implants Res 1999; 10: 111-119

[38] Roos-Jansaker AM, Persson GR, Lindahl C et al. Surgical treatment of peri-implantitis using a bone substitute with or without a resorbable membrane: a 5-year follow-up. J Clin Periodontol 2014; 41: 1108-1114

[39] Daugela P, Cicciù M, Saulacic N. Surgical regenerative treatments for peri-implantitis: meta-analysis of recent findings in a systematic literature review. J Oral Maxillofac Res 2016; 7: e15

[40] Schwarz F, Hegewald A, John G et al. Four-year follow-up of combined surgical therapy of advanced peri-implantitis evaluating two methods of surface decontamination. J Clin Periodontol 2013; 40: 962-967

[41] de Waal YC, Raghoebar GM, Huddleston Slater J] et al. Implant decontamination during surgical peri-implantitis treatment: a randomized, double-blind, placebo-controlled trial. J Clin Periodontol 2013; 40: 186-195

[42] Regev E, Shiff JS, Kiss A et al. Internal fixation of mandibular angle fractures: a meta-analysis. Plast Reconstr Surg 2010; 125: $1753-1760$

[43] Danda AK. Comparison of a single noncompression miniplate versus 2 noncompression miniplates in the treatment of mandibular angle fractures: a prospective, randomized clinical trial. J Oral Maxillofac Surg 2010; 68: 1565-1567

[44] Hermund NU, Hillerup S, Kofod T et al. Effect of early or delayed treatment upon healing of mandibular fractures: a systematic literature review. Dental Traumatol 2008; 24: 22-26 
[45] Schaefer EH 4th, Caterson E]. Antibiotic selection for open reduction internal fixation of mandible fractures. J Craniofac Surg 2013; 24: 85-88

[46] Ghanem WA, Elhayes KA, Saad K. The management of unstable oblique infected mandibular fractures with a $2.3 \mathrm{~mm}$ mandibular osteosynthesis reconstruction bone plate. J Craniomaxillofac Surg 2011; 39: 600-605

[47] Knepil G], Loukota RA. Outcomes of prophylactic antibiotics following surgery for zygomatic bone fractures. J Craniomaxillofac Surg 2010; 38: 131-133

[48] Maloney PL, Lincoln RE, Coyne CP. A protocol for the management of compound mandibular fractures based on the time from injury to treatment. J Oral Maxillofac Surg 2001; 59: 879-884

[49] Lee HB, Oh JS, Kim SG et al. Comparison of titanium and biodegradable miniplates for fixation of mandibular fractures. J Oral Maxillofac Surg 2010; 68: 2065-2069

[50] Cahill T] 3rd, Gandhi R, Allori AC et al. Hardware removal in craniomaxillofacial trauma: a systematic review of the literature and management algorithm. Ann Plast Surg 2015; 75: 572-578

[51] Zanotti B, Zingaretti N, Verlicchi A et al. Cranioplasty: review of materials. J Craniofac Surg 2016; DOI: 10.1097/ scs. 0000000000003025

[52] Shah AM, Jung H, Skirboll S. Materials used in cranioplasty: a history and analysis. Neurosurg Focus 2014; 36: E19
[53] Kimchi G, Stlylianou P, Wohl A et al. Predicting and reducing cranioplasty infections by clinical, radiographic and operative parameters - a historical cohort study. J Clin Neurosci 2016; 34: $182-186$

[54] Young RF, Lawner PM. Perioperative antibiotic prophylaxis for prevention of postoperative neurosurgical infections. A randomized clinical trial. J Neurosurg 1987; 66: 701-705

[55] Walcott BP, Kwon CS, Sheth SA et al. Predictors of cranioplasty complications in stroke and trauma patients. J Neurosurg 2013; 118: 757-762

[56] Malcolm JG, Rindler RS, Chu JK et al. Complications following cranioplasty and relationship to timing: a systematic review and meta-analysis. J Clin Neurosci 2016; 33: 39-51

[57] Yadla S, Campbell PG, Chitale R et al. Effect of early surgery, material, and method of flap preservation on cranioplasty infections: a systematic review. Neurosurgery 2011; 68: 1124 1129

[58] Conen A, Fux CA, Vajkoczy P et al. Management of infections associated with neurosurgical implanted devices. Expert Rev Anti Infect Ther 2017; 15: 241-255

[59] Brown EM, Edwards R], Pople IK. Conservative management of patients with cerebrospinal fluid shunt infections. Neurosurgery 2008; 62 (Suppl. 2): S661-S669

Bibliografie

DOI https://doi.org/10.1055/s-0043-102341

Online-publiziert 15.05.2017 | OP-JOURNAL 2017; 33: 149158 @ Georg Thieme Verlag KG Stuttgart · New York ISSN 0178-1715 